\title{
ELECCIÓN DE MODALIDAD EDUCATIVA EN LA ENSEÑANZA MEDIA Y SU ROL EN LA POSTULACIÓN A LAS UNIVERSIDADES DEL $\mathrm{CRUCH}^{1}$
}

\author{
Ximena Catalán ${ }^{2}$
}

RESUMEN

Aun cuando está enfocada en la preparación para el mundo laboral, crecientemente la enseñanza media técnico-profesional (EMTP) se ha constituido como una vía de acceso a la educación superior en Chile. En este contexto, esta investigación aporta conocimiento acerca de las trayectorias educacionales de los estudiantes que cursan la EMTP, abordando dos principales ámbitos: la preferencia por esta modalidad educativa en la enseñanza media y la postulación a la universidad de los jóvenes que la cursaron durante sus estudios secundarios. Con tal objetivo, se evaluó la asociación entre una serie de variables relacionadas con el habitus y la preferencia por cursar la EMTP. Asimismo, se estudió si haber seguido dicha modalidad educativa afectaba la probabilidad de postulación de los jóvenes a las universidades del Consejo de Rectores de las Universidades Chilenas (CRUCH), controlando por variables socioeconómicas y académicas. Los resultados de esta investigación respaldan la relevancia de las predisposiciones sociales sobre las trayectorias educacionales, destacando el rol que tendría la modalidad educativa de la enseñanza media en la reproducción de las diferencias sociales de los estudiantes.

Palabras clave: educación universitaria, enseñanza técnico-profesional, oportunidades educacionales.

\section{SELECTION OF HIGH SCHOOL MODALITY AND ITS ROLE IN APPLYING TO THE CRUCH UNIVERSITIES}

\begin{abstract}
Even though vocational education at the secondary level is focused on preparing students for the work world, in Chile, it has turned into a way to enter higher education. In this context, this research aims to increase the knowledge about educational trajectories of students in vocational education at the secondary level, by addressing two main areas: factors related to a preference for vocational secondary school and the future choice of these students to apply to a university. This investigation, thus, studied the association between a set of variables related to habitus and the preference for studying vocational education at the secondary level. Additionally, it investigated if having studied this modality in secondary education affects the probability that these students will apply to the universities of the Consejo de Rectores de las Universidades Chilenas (CRUCH, Board of Regents of Chilean Universities), after controlling for academic and socioeconomic variables. This investigation's results support the relevance of social predispositions on educational trajectories and highlight the role that the modality of secondary level education would have on the reproduction of the students' social differences.
\end{abstract}

Keywords: technical and vocational education, university education, educational opportunities.

1 Esta investigación contó con apoyo financiero del programa de becas de CONICYT y de la Fundación Volcán Calbuco.

2 Facultad de Educación, Pontificia Universidad Católica de Chile, Santiago, Chile. Contacto: xrcatala@uc.cl 


\section{Introducción}

En Chile, la enseñanza secundaria se encuentra diferenciada en dos modalidades educativas: educación media técnico-profesional (EMTP) y educación media científico-humanista $(\mathrm{EMCH})^{3}$. Mientras esta última tiene como objetivo preparar a los estudiantes en conocimientos generales que les permitan continuar estudios superiores, la EMTP busca generar habilidades para la inserción laboral de sus alumnos.

Luego de un aumento sostenido durante los años noventa, al año 2012 un 43\% de la matrícula de tercer y cuarto año de enseñanza secundaria estaba matriculado en la EMTP (Centro de Estudios del Ministerio de Educación de Chile, Mineduc, 2013). Según cifras del año 2010, esta modalidad se imparte en 946 establecimientos educativos y alrededor de un tercio de ellos ofrece, además, la modalidad científico-humanista, en los llamados establecimientos polivalentes (EMPV) (Mineduc, 2011).

Existe una alta segmentación socioeconómica en la modalidad educativa que los estudiantes cursan durante su enseñanza media: $60 \%$ de quienes provienen del quintil de menores ingresos cursa EMTP, cifra que apenas alcanza un 12\% en el caso de los jóvenes provenientes del quintil de mayores ingresos (Larrañaga, Cabezas y Dussaillant, 2013).

Pese a ser una educación orientada al trabajo y, por lo tanto, menos intensiva en términos de preparación académica para continuar estudios terciarios, dentro del grupo de estudiantes que asiste a la EMTP existe un creciente interés por realizar estudios superiores y postergar la inserción laboral (Farías y Sevilla, 2012; Sepúlveda,

3 La diferenciación entre modalidades de enseñanza en el sistema educativo chileno, hasta fines de la década de los noventa, ocurría al inicio de la educación secundaria (primero medio). Para que los jóvenes tuviesen una formación general similar durante, al menos, los diez primeros años de enseñanza formal, la Reforma Curricular de 1998 estableció que la elección se realizara dos años más tarde, al inicio de tercer año de enseñanza secundaria (Mineduc, 2011). Sin embargo, entre quienes se cambian de colegio para cursar estudios secundarios, la mayoría lo hace cuando está pasando a primero medio y solo alrededor de un tercio de los colegios que ofrecen EMTP cuentan, además, con la modalidad científicohumanista. Por lo tanto, pese a la disposición legal, la elección de modalidad educativa se haría la mayoría de las veces a fines de octavo año de enseñanza básica. 
Ugalde y Campos, 2009). Asimismo, aunque las familias de quienes asisten a la EMTP tienen menores expectativas de que sus hijos asistan a la educación superior en comparación con quienes tienen hijos en la EMCH, la proporción de padres de estudiantes cursando la EMTP que aspira a que estos continúen estudios en la educación terciaria es alta (Centro de Estudios del Mineduc, 2013). No obstante, los estudiantes provenientes del sector EMTP se encuentran con grandes dificultades para ingresar a una universidad, incluyendo la falta de alineación entre el currículo cursado y los contenidos medidos por la Prueba de Selección Universitaria (PSU), que los lleva a tener resultados sistemáticamente más bajos en dicha evaluación (Farías y Carrasco, 2012; Pearson, 2013).

El menor nivel socioeconómico y la menor preparación académica de los estudiantes que cursan la EMTP quedan reflejados en sus trayectorias educacionales. En primer lugar, existe una menor proporción de egresados de la EMTP que ingresa a la educación superior (41\% versus un $67 \%$ en el grupo de egresados de la EMTP) y entre quienes lo hacen, solo un $20 \%$ ingresa al año siguiente de haber egresado de la educación secundaria, mientras que en el grupo proveniente de la EMCH esta cifra alcanza a alrededor de un 50\% (Larrañaga et al., 2013). Adicionalmente, mientras que alrededor de un $62 \%$ de quienes provienen de la EMTP se matricula en instituciones técnicas (CFT e IP), un 70\% de quienes acceden a la educación superior habiendo egresado de la $\mathrm{EMCH}$ se matricula en universidades, y solo alrededor de un $8 \%$ de los alumnos de la EMTP asiste a las universidades acreditadas por seis o siete años, en comparación con un $22 \%$ de quienes provienen de la EMCH (Larrañaga et al., 2013).

Pese a las inequidades mencionadas anteriormente, durante los últimos años, la EMTP se ha instaurado como una vía relevante para el ingreso a la educación superior en Chile, lo que se ha reflejado en un creciente interés por abordar el rol de la modalidad educativa cursada en la enseñanza media en las trayectorias educacionales de los jóvenes (Farías, 2013; Farías y Carrasco, 2012; Farías y Sevilla, 2012). En esta misma línea, la siguiente investigación busca aportar al conocimiento acerca de las trayectorias a la educación superior 
de los estudiantes que cursaron la EMTP, utilizando como marco de referencia el concepto de habitus (Bourdieu, 1987). En específico, la investigación indagó la relevancia que tiene un grupo de variables relacionadas con el habitus familiar respecto de la educación en la modalidad para la enseñanza media preferida por las familias para sus hijos. Asimismo, se exploraron las diferencias según modalidad educativa del establecimiento de enseñanza media del estudiante en la postulación a las universidades del $\mathrm{CRUCH}$.

\section{Revisión de la literatura}

\subsection{Elección de modalidad educativa en la enseñanza secundaria}

La elección del establecimiento de educación secundaria y de la modalidad educativa a seguir por parte de los estudiantes en este nivel educativo han sido poco estudiadas en Chile. Dentro de las escasas investigaciones que han abordado esta elección, se encuentra el estudio desarrollado por De Iruarrizaga (2009) quien encontró, a partir de un modelo logit, que tanto el ingreso familiar como la habilidad académica son relevantes en esta decisión, siendo aquellos estudiantes con menores ingresos y menor habilidad académica quienes en mayor medida optan por la EMTP. Apoyando estos resultados, el estudio de Larrañaga et al. (2013) mostró que existe una mayor relevancia del factor socioeconómico que del académico en la elección de EMTP: el joven con mejor desempeño del quintil de menores ingresos tiene 2,75 veces mayor probabilidad de ingresar a la EMTP que el alumno con peor desempeño del quintil de mayor ingreso. Por su parte, Raczynski, Hernández, Rejevic y Roco (2011), a partir de una metodología cualitativa, encontraron que existe una valoración positiva de la EMTP por parte de las familias en contextos vulnerables, quienes ven en este tipo de modalidad educativa una mayor seguridad para el futuro laboral de sus hijos. Otros aspectos relevantes en la elección de la EMTP son: ser hombre, tener padres con un menor nivel educativo y la existencia de una mayor oferta académica de este tipo de establecimientos en la comuna de residencia del estudiante (De Iruarrizaga, 2009). 
Por su parte, al estudiar el proceso de elección de escuela en la educación básica, la investigación de Elacqua y Fabrega (2004) encontró que las familias de menor nivel socioeconómico le dan una mayor importancia a los aspectos prácticos en la elección del establecimiento (cercanía al hogar o al trabajo de los padres, su costo, el horario de clases, etc.) que a los académicos. Sin embargo, la investigación de Flores y Carrasco (2013) hace hincapié en que son las restricciones presupuestarias que enfrentan las familias y la oferta local de establecimientos, las que generan estas diferencias en la valoración de sus distintos atributos o funciones. En este marco, la preferencia por un colegio con EMTP podría entenderse como una elección anclada en el nivel socioeconómico de las familias y en su estructura local de oportunidades, que las llevan a considerar en un segundo plano los aspectos académicos.

\subsection{La transición a la educación superior}

En Chile, si bien no ha existido un cuerpo teórico importante para el estudio de las trayectorias educacionales, algunas investigaciones han abordado los determinantes del acceso a la educación superior, destacando la importancia de aspectos socioeconómicos, académicos y de ayuda financiera (Acuña, Makovec \& Mizala, 2010; Canales, 2015; Meneses y Blanco, 2010; Solís, 2012). En países como Estados Unidos, en cambio, han surgido diversas perspectivas conceptuales para comprender esta transición que tienen como rasgo común comprender el tránsito a la educación superior como un proceso de largo plazo, que comienza a desarrollarse desde antes del ingreso a la educación secundaria y en el que confluye una serie de dimensiones (Adelman, 2006; Bell, Rowan-Kenyon \& Perna, 2006; Cabrera \& La Nasa, 2001; Hossler, Braxton \& Coopersmith, 1989; McDonough, 1997; Perna, 2006; Perna, Rowan-Kenyon, Bell, Thomas \& Li, 2008). Hossler et al. (1989) destacan tres principales fases en el proceso de ingresar a la universidad: adquirir las competencias mínimas para la universidad (incluyendo aspectos como promedios y ranking de notas, puntajes en pruebas estandarizadas, etc.); graduarse de la educación media; y postular a una universidad. Durante dichas fases, el estudiante va desarrollando predisposiciones, realiza búsquedas y finalmente toma decisiones respecto de la educación superior. 
Perna (2006), utiliza el concepto de habitus de Bourdieu para explicar la toma de decisiones en el nivel individual, aunque comprendiendo dicho concepto dentro de una serie de capas que determinan y dan espacio a dicha expresión.

Utilizando el enfoque desarrollado por Hossler et al. (1989), la investigación de Cabrera y La Nasa (2001) mostró que un 71\% de los jóvenes de menor nivel socioeconómico, en comparación con un $30 \%$ de estudiantes de mayores recursos, no contaba con las calificaciones mínimas para la educación superior, un aspecto clave en la continuación de estudios terciarios. Asimismo, las pruebas estandarizadas de selección han mostrado una importante brecha entre estudiantes de distinto nivel socioeconómico (Organización para la Cooperación y el Desarrollo Económicos, OCDE, 2009; Zwick \& Grief Greene, 2007). Al respecto McDonough (1988) sostiene que los estudiantes de familias de menor nivel socioeconómico se involucran con la educación superior de manera tardía al compararlos con sus pares de mayor nivel socioeconómico, quienes ya desde la enseñanza primaria preparan su ingreso a la universidad, por ejemplo, tomando cursos relevantes para los estudios universitarios.

Algunos estudios han enfatizado que las diferencias según nivel socioeconómico en el acceso a la educación superior no pueden ser explicadas exclusivamente a partir de los aspectos académicos de los estudiantes. Según el estudio de Cabrera y La Nasa (2001), solo un $65 \%$ de los jóvenes con un alto grado de preparación académica perteneciente a un nivel socioeconómico bajo postuló a la universidad, 22 puntos porcentuales por debajo de estudiantes con una preparación similar provenientes de familias con un alto nivel de ingreso. En la misma línea, Canales (2015) encontró, para el caso chileno, que las diferencias por nivel socioeconómico en la decisión de postular a las universidades del CRUCH no podían ser explicadas solo por aspectos académicos.

La postulación a la universidad es un proceso multidimensional en el que entran en juego al mismo tiempo la habilidad académica, el conocimiento del sistema de la educación superior y de la oferta de programas, la disponibilidad de recursos financieros para continuar 
estudios, el grado de seguridad del estudiante sobre sus aptitudes, etc. (Cabrera \& La Nasa, 2001). Los jóvenes provenientes de entornos desaventajados tienen un menor grado de familiaridad e información acerca de las características del sistema de educación superior, al contar con padres que no asistieron a instituciones terciarias e ir a escuelas con poco apoyo en relación con el proceso de postulación (Bell et al., 2006; McDonough, 1997). Entre quienes sí postulan a la universidad, aspectos relacionados con las características institucionales podrían llevar a estudiantes de desempeño similar a preferir determinadas instituciones por sobre otras, a partir del grado de cercanía que perciban entre la institución y sus propias características y aspiraciones (Karen, 1988).

\section{Marco teórico}

En el ámbito de la investigación respecto de decisiones y resultados académicos, el concepto de capital cultural (Bourdieu, 2001) ha sido frecuentemente utilizado (Dumais, 2002). No obstante, este concepto es solo uno de los pilares en la teoría social de Bourdieu, siendo también fundamentales las nociones de campo y habitus (Bourdieu, 1984).

El habitus refiere a las disposiciones generadas a partir de una posición específica en la estructura social, que influencian el actuar de los individuos (Bourdieu, 1987). La introducción de este concepto concilia dos perspectivas opuestas en el estudio de la realidad social: el subjetivismo, que comprende al sujeto como un ser que elige libremente, y el objetivismo, que lo concibe como un mero soporte de la estructura social. Bourdieu plantea que los sujetos, aunque no son libres en sus decisiones (ya que el habitus, el principio regulador de todas las elecciones, no se elige), tampoco están obligados a una elección u otra, sino que más bien, tienen a la mano un conjunto de elecciones o prácticas posibles. En la misma línea, la introducción del concepto de racionalidad práctica (Bourdieu, 1997) emerge como una crítica a las nociones abstractas de racionalidad, proponiendo comprender las racionalidades prácticas de los sujetos en el contexto de sus diferentes habitus o esquemas de acción, los que emergen en condiciones sociales y materiales particulares, restringiendo, pero sobre todo, posibilitando la acción. 
Según el marco general del habitus y de la teoría de la práctica de Bourdieu, aspectos como la forma en que los sujetos comprenden la educación y las funciones que asocian a ella, podrían ser explicados a partir de su habitus particular. Este habitus los llevará, entre otras cosas, a valorar ciertas dimensiones de la educación por encima de otras, por ejemplo, a priorizar los aspectos prácticos de los establecimientos educacionales (como la ubicación o el precio) por sobre los académicos. En el contexto de estas valoraciones las familias contemplarán, dentro de sus posibilidades, colegios con determinadas características a la hora de escoger un establecimiento educacional y, finalmente, optarán por alguno de ellos.

Por otra parte, las expectativas o "esperanzas subjetivas" (Bourdieu, 2006) que las familias depositan en sus hijos en el ámbito educativo, también pueden ser comprendidas en el marco general de su habitus. Estas expectativas tienen que ver, entre otras cosas, con las trayectorias académicas que se consideran razonables para los estudiantes: terminar o no la educación secundaria y en qué tipo de establecimiento, ingresar a la educación superior, incluyendo el tipo de institución o programa académico escogido, entre otros aspectos. El sentido práctico llevaría a la adaptación, aunque no a la determinación, de las esperanzas subjetivas a las condiciones objetivas.

De esta forma, tanto las características valoradas de la educación como las expectativas que tienen las familias de los estudiantes en relación con sus estudios están relacionadas con su habitus. Las familias de entornos desaventajados, que en general valoran en mayor medida los aspectos prácticos de los establecimientos y que tienden a tener menores expectativas respecto del futuro académico de sus hijos, tendrán un habitus menos orientado hacia la educación superior y, por lo tanto, puede hipotetizarse que serán más proclives a optar por establecimientos EMTP para que sus hijos cursen estudios secundarios. Asimismo, un habitus menos orientado hacia los estudios superiores limitará, aunque no imposibilitará, la disposición de los jóvenes a seguir tracks académicos más demandantes, como por ejemplo, ingresar a la universidad. Del mismo modo, su habitus generará un rango específico de instituciones que considerarán en caso de continuar sus estudios superiores. De esta forma, es posible sostener 
que a partir de su habitus, estos jóvenes tendrán menos probabilidades de postular a las universidades y, en especial, a las universidades más selectivas del país.

En la presente investigación, el habitus se abordará indirectamente a través de variables que, se sostiene, están relacionadas con dicho concepto, en específico, las funciones asociadas a la educación y las expectativas educativas que tienen las familias en los estudiantes, a través de cuatro variables empíricas utilizadas para la predicción de la preferencia de modalidad educativa para la enseñanza media. Nuevamente es necesario aclarar que al usar estas variables no se pretende realizar una medición del habitus propiamente tal, sino de algunas de sus manifestaciones ${ }^{4}$.

Siguiendo a Hossler et al. (1989), estas variables se estudiarán en la etapa de "predisposición" a la educación superior, la que habitualmente se desarrolla en los últimos años de la enseñanza primaria y que refiere al periodo en que los estudiantes comienzan a pensar si quieren continuar estudios terciarios. Es importante mencionar que, aun cuando se teoriza que hay una relación entre el habitus y la decisión de postulación a la universidad, dicho vínculo no fue abordado directamente en este estudio, debido a limitaciones con las fuentes de información. No obstante, se considera que las variables relacionadas con el habitus incluidas en la investigación afectan la postulación a la universidad a través de la modalidad educativa escogida para la enseñanza secundaria.

De esta forma, la elección de modalidad educativa en la educación secundaria es comprendida en esta investigación como una decisión clave dentro de las trayectorias educativas de los jóvenes en Chile. Dicha decisión es tomada por las familias en un contexto situado que refiere a una posición específica dentro del campo educativo, determinada por su nivel de acumulación de los diversos capitales y por una disposición hacia la educación que se

4 Abordar empíricamente el habitus resulta desafiante al tratarse de un concepto eminentemente abstracto ("disposiciones" o "esquemas de acción").Conceptos como el de "milieu" (Vester, 2003) son sin duda mucho más fieles a la naturaleza profunda y relacional del habitus, pero escapan a la información utilizada para esta investigación. 
estructura a partir de esta posición. Al mismo tiempo, la modalidad educativa cursada durante la educación media abre un camino específico hacia la educación superior, dadas las diferencias entre modalidades en lo referente a aspectos académicos, expectativas y el grado de familiarización con la educación superior, lo cual afectaría las probabilidades que tienen los jóvenes de postular a la universidad.

La Figura 1 muestra de manera gráfica el marco conceptual considerado en esta investigación, incluyendo las principales variables utilizadas. En el diagrama se muestran tanto las relaciones estudiadas empíricamente en la investigación como aquellas que si bien son relevantes, no fueron abordadas (líneas punteadas). Al igual que el modelo presentado por Perna et al. (2008), se presenta una capa relacionada con aspectos de la política educacional, entendida como el contexto general en el que se toman las decisiones educativas.

Figura 1. Esquema conceptual usado en esta investigación

\section{Ámbito de la política educativa \\ Estructura de diferenciación en el sistema educativo / Características del sistema de admisión a la educación superior}

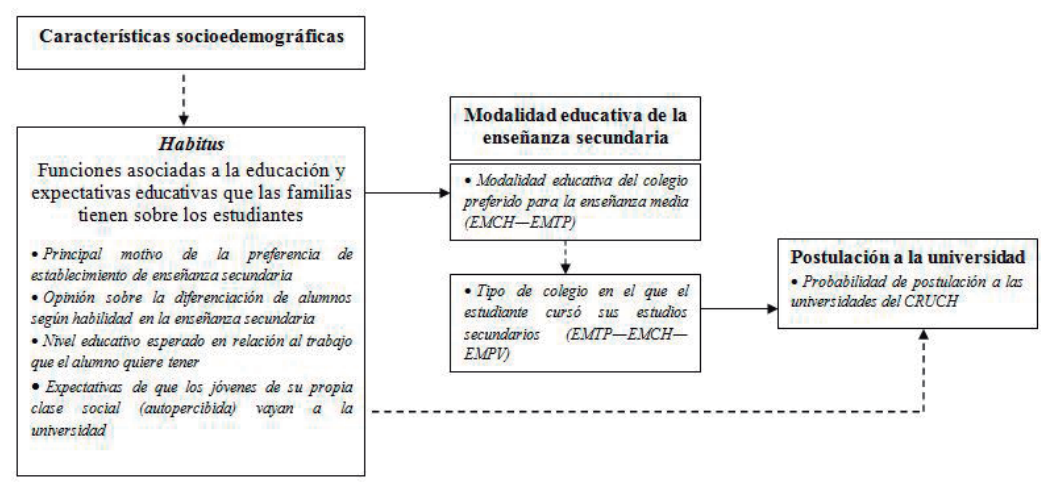

Fuente: Elaboración propia sobre la base de Perna (2006) y Perna et al. (2008).

\section{Metodología}

\subsection{Objetivos}

El objetivo general de esta investigación fue indagar en los factores que determinan la modalidad educativa preferida por las familias para la enseñanza secundaria de sus hijos y en la relevancia que tiene 
la modalidad educativa del establecimiento de enseñanza media del estudiante en su postulación a las universidades del $\mathrm{CRUCH}$.

Por su parte, los objetivos específicos de investigación que surgieron en este contexto son: indagar la relevancia que tienen las funciones y las expectativas asociadas a la educación por parte de las familias (variables relacionadas con el habitus) en la modalidad educativa de enseñanza secundaria preferida por estas para sus hijos; y explorar el rol que tiene la modalidad educativa del establecimiento de enseñanza media del estudiante en su decisión de postular a las universidades del CRUCH.

Cabe señalar que se utilizó la postulación a las universidades del CRUCH al ser las únicas que, al momento de la investigación, contaban con un sistema de admisión centralizado. Además, se privilegió esta fuente porque permitía conocer la familiaridad de los estudiantes de la EMTP con universidades que: reciben financiamiento estatal directo y tienen mayor disponibilidad de beneficios estudiantiles por parte del Estado, muchos de los cuales están orientados exclusivamente en este tipo de instituciones (Comisión de financiamiento estudiantil para la educación superior, 2012); cuentan con mayores tasas de persistencia y titulación de sus estudiantes (Consejo Nacional de Educación, CNED, 2010) y son las universidades de mayor tradición y prestigio del sistema de la educación superior en Chile (OCDE, 2009). De esta forma, aun cuando los jóvenes de la EMTP puedan tener la preferencia por continuar estudios superiores en otro tipo de instituciones, o incluso de no continuarlos, es pertinente conocer la cercanía que existe entre ellos y las instituciones del CRUCH.

La hipótesis que guió esta investigación fue que los condicionamientos socioeconómicos, incluyendo las funciones y expectativas asociadas a la educación, son fundamentales para las familias a la hora de preferir una modalidad educativa para la enseñanza secundaria de sus hijos. Además, se sostiene que la modalidad educativa del colegio de enseñanza media es una pieza clave en la configuración de las oportunidades que tienen los jóvenes en su camino hacia la educación superior, en especial, de acceder a las universidades de mayor tradición en el país. En este sentido, se 
hipotetiza que la elección de modalidad educativa para la enseñanza secundaria podría estar asociada con la reproducción de las diferencias de origen entre estudiantes de distinto nivel socioeconómico.

\subsection{Fuentes de información y muestra}

Este estudio utilizó dos principales fuentes de información: la encuesta PASC II Ola 1 y la información de postulación a las universidades del CRUCH entregada por el Demre (Departamento de Evaluación, Medición y Registro de la Universidad de Chile). Se trata de dos conjuntos de información independientes, con distintas muestras, por lo que no se pudo hacer un seguimiento de cohorte.

\subsubsection{Encuesta PASC II Ola 1}

Para indagar en los factores que influyen en la preferencia de modalidad educativa para la enseñanza media, se utilizó información de la investigación Parents and school choice: Family strategies, segregation and school policies in Chilean and Finnish primary schooling (PASC), en particular, de la encuesta realizada el año 2011 (primera ola), la cual consideraba preferencias de establecimientos (y no postulación efectiva). El proyecto PASC-AKA tuvo como objetivo indagar en distintas consideraciones acerca de la elección de escuela tanto para primer año de enseñanza básica, como para primer año de enseñanza media, utilizándose en esta investigación solo la encuesta relacionada con la elección de establecimiento para la enseñanza secundaria (PASC II).

PASC II es una encuesta estratificada según los cinco grupos socioeconómicos de la clasificación Adimark (ABC1, C2, C3, D y E), centrada en familias de la Región Metropolitana con estudiantes en sexto año básico que debían cambiarse de institución para cursar estudios secundarios. La muestra de esta encuesta corresponde a 418 casos, siendo representativa de la población objetivo del estudio. La distribución por nivel socioeconómico indica una mayor presencia de familias de nivel socioeconómico D (44,3\%) y C3 (34,5\%), con una menor representación de los segmentos E (9,8\%), C2 (9,3\%) y ABC1 $(2,2 \%)$. Al respecto, cabe destacar que los estudiantes provenientes del nivel socioeconómico $\mathrm{ABCl}$ se encuentran subrepresentados en 
relación con su total a nivel de la región, dadas las características de la población objetivo de esta investigación ${ }^{5}$. La muestra cuenta con un $51,2 \%$ de alumnos de sexo masculino y, del total de casos, a un $85 \%$ (355) se les pudo asignar una modalidad educativa deseada para la enseñanza secundaria 6 .

\subsubsection{Proceso de postulación 2011 a las universidades del CRUCH} (Demre)

Para obtener información respecto de la postulación a las universidades del CRUCH se utilizaron bases de datos del Demre, organismo encargado de administrar el proceso de admisión centralizado en estas universidades ${ }^{7}$. Se utilizó como universo de estudio el grupo de postulantes inscritos para el proceso de admisión 2011 a las universidades del CRUCH que pertenecían a la generación del año, es decir, que habían egresado de la enseñanza secundaria el año inmediatamente anterior $(N=211.691)$, cifra que corresponde a un $73,2 \%$ del total de inscritos de dicho proceso (Demre, 2011). Esto se hizo para contar con una muestra de estudiantes que, habiendo salido recién del colegio, se enfrentaban por primera vez al proceso de postulación al CRUCH. Dentro del total de inscritos de la generación, un $84,7 \%(n=179.248)$ rindió la PSU ${ }^{8}$ ese año y un 33,3\% $(n=59.609)$ de quienes rindieron postuló a alguna de las universidades tradicionales $^{9}$ (ver Tabla 1).

5 Esto sucede porque en el nivel socioeconómico alto la mayoría de los establecimientos cuenta con enseñanza básica y media y, por lo tanto, quienes asisten a ellos, no cumplen con los requisitos para ser parte de la investigación, ya que no tienen que cambiarse de colegio para cursar estudios secundarios. Al revisar datos del Simce, los colegios particulares pagados representan cerca de un 12\% en el total general de colegios de la Región Metropolitana, mientras que esta cifra baja a alrededor de un 3\% al considerar los colegios particulares pagados que ofrecían solo enseñanza básica. Esta situación podría traer consecuencias en los resultados de este estudio, al contar con menos estudiantes de nivel socioeconómico alto en la muestra, quienes además podrían presentar características muy específicas (Fuente: cálculos propios sobre la base de la información del Simce año 2012).

6 En general los modelos de regresión implementados cuentan con un total de casos cercano a ese valor, dependiendo de la disponibilidad del resto de las covariables utilizadas.

7 Desde la admisión 2012, además participan ocho universidades privadas creadas con posterioridad a 1981 .

8 Se considera "rendir la PSU" contestar al menos las pruebas de Matemáticas y Lenguaje y Comunicación, ya que ambas son indispensables para postular válidamente a las universidades del CRUCH.

9 Incluyendo postulaciones válidas e inválidas, es decir, independientemente de si estas fueron consideradas admisibles por las universidades. 
Tabla 1. Participantes en las distintas etapas del proceso de postulación a las universidades del CRUCH (admisión 2011)

\begin{tabular}{l|c}
\hline Proceso 2011 & Total \\
\hline Matrícula $4^{0}$ medio 2010* & 266.862 \\
\hline Inscritos del proceso & 211.691 \\
\hline Rinden la PSU & 179.248 \\
\hline (\% rinde PSU sobre total inscritos) & $84,7 \%$ \\
\hline Postulan al CRUCH & 59.609 \\
\hline (\% postulan al CRUCH sobre total que rinde) & $33,3 \%$ \\
\hline
\end{tabular}

Fuente: Elaboración propia sobre la base de información Demre.

*Según anuario Demre 2011.

El modelo de regresión implementado para la decisión de postular a las universidades del $\mathrm{CRUCH}$ ocupó como muestra a quienes rindieron la PSU, requisito imprescindible para postular a estas universidades mediante el sistema centralizado, variando el total de casos según la disponibilidad del resto de las variables consideradas. En el análisis solo se consideró a quienes pertenecían a establecimientos municipales y particulares subvencionados ( $n=146.499$ ), con el objetivo de que los grupos de modalidad educativa fueran más comparables entre sí, tomando en cuenta que en el sector particular pagado no existen colegios con EMTP o EMPV.

\subsection{Variables principales}

En esta sección se presenta una descripción de las variables de mayor interés de la investigación: modalidad educativa en la enseñanza media, variables relacionadas con el habitus y postulación a las universidades del CRUCH.

\subsubsection{Modalidad educativa en la enseñanza media}

La variable central de esta investigación es la modalidad educativa en la enseñanza secundaria, que será utilizada como variable a explicar en el caso del primer objetivo específico, y como variable predictora en el caso del segundo objetivo específico. En el primer caso, la variable se obtuvo vinculando la primera preferencia de establecimiento para la enseñanza media mencionada en la encuesta PASC II con la modalidad educativa de ese establecimiento según los registros de Demre. 
La variable modalidad educativa fue operacionalizada diferencialmente para los dos objetivos específicos. Para el primero, se utilizó como una variable dicotómica indicando si el colegio mencionado como primera preferencia por las familias contaba con EMTP o solo ofrecía EMCH. Esta dicotomización se hizo para contar con grupos de comparación más grandes. En relación con el proceso de postulación a las universidades del $\mathrm{CRUCH}$, la modalidad educativa se incluyó a través de dos variables dicotómicas, EMTP (colegios solo ofreciendo enseñanza media técnico-profesional) y EMPV (establecimientos de educación media polivalente, es decir, que ofrecen ambas modalidades educativas), siendo los establecimientos $\mathrm{EMCH}$ la categoría de referencia. Cabe destacar que esta variable refiere al colegio, independientemente de si el alumno siguió una modalidad educativa u otra, lo que se hizo para dar cuenta de un posible efecto del establecimiento sobre la probabilidad de postulación de los estudiantes.

\subsubsection{Postulación a las universidades del CRUCH}

La variable a predecir en el caso del segundo objetivo específico es la postulación a cualquiera de las universidades del CRUCH, la cual se realiza online de manera centralizada y los postulantes del proceso de admisión 2011 podían marcar hasta ocho preferencias. Cabe destacar que el proceso de postulación se realiza luego de que los jóvenes conocen sus puntajes en la PSU, por lo que pueden comparar su puntaje ponderado (según los requisitos de cada programa de estudios) con los puntajes de corte de los años anteriores para la misma carrera, conociendo de antemano sus probabilidades aproximadas de ser seleccionados. Por lo tanto, pese a que la postulación refleja en gran medida las preferencias del estudiante, la decisión de postulación también podría estar influenciada por otros aspectos, como la certeza de ser admitido en una determinada carrera y universidad.

\subsubsection{Variables relacionadas con el habitus}

Como se mencionó anteriormente, las variables relacionadas con el habitus que fueron medidas en la investigación refieren a las funciones asociadas a la educación y las expectativas educativas que las familias tienen de los estudiantes. Las variables empíricas utilizadas son: el 
motivo principal en la preferencia del establecimiento educativo para la enseñanza secundaria ${ }^{10}$; la opinión acerca de la diferenciación por habilidad académica en la educación; el nivel educativo que la familia espera que el alumno alcance (dado el trabajo que quiere desempeñar $)^{11}$; y la creencia de que, en la clase social autopercibida, los jóvenes asisten a la universidad. Este grupo de variables puede asociarse a una disposición por parte de las familias de que el estudiante continúe o no estudios universitarios. En la Tabla 2 se presenta la relación esperada entre las variables específicas utilizadas para medir el habitus, la disposición a cursar estudios universitarios y la variable de resultado (modalidad educativa preferida para los estudios secundarios) ${ }^{12}$.

10 Existen 21 categorías de respuesta para esta pregunta, las cuales, para efectos de esta investigación, pueden ser divididas en cinco principales áreas: 1) motivos relacionados con el acceso a la universidad (variable considerada en los modelos); 2) motivos relacionados con aspectos sociales (conocimiento del colegio a partir de la propia experiencia o de familiares o amigos, búsqueda de un nivel social o religión similar al propio, etc.); 3) motivos relacionados con aspectos laborales (buen nivel de inglés, generación de una red de contactos, etc.); 4) motivos relacionados con aspectos de docencia (los profesores son de excelente calidad humana o profesional, colegios pequeños, etc.); 5) motivos prácticos (cercanía con el hogar, seguridad, disciplina, etc.). También está la categoría "otros", en la que se permitió una respuesta abierta y además se integraron las categorías "son colegios que se orientan a las artes y/o el deporte" y "son colegios orientados específicamente a formar buenos ciudadanos" al no poder clasificarse en ninguna de las categorías anteriores.

11 Estos niveles consideran: básica, media científico-humanista, técnica de nivel superior, universitaria y posgrado.

12 Las variables relacionadas con el habitus incluidas en la investigación en algunos casos contemplaban más de dos categorías de respuesta, pero fueron dicotomizadas con el objetivo de enfatizar las dimensiones relevantes para la investigación. 
Tabla 2. Variables relacionadas con el habitus y su relación con la preferencia por la modalidad EMTP

\begin{tabular}{|c|c|c|c|}
\hline $\begin{array}{l}\text { Variables } \\
\text { relacionadas con el } \\
\text { habitus }\end{array}$ & Categorías & $\begin{array}{l}\text { Disposición a } \\
\text { cursar estudios } \\
\text { universitarios }\end{array}$ & $\begin{array}{l}\text { Relación con la variable de } \\
\text { resultado }\end{array}$ \\
\hline $\begin{array}{l}\text { Motivo } \\
\text { Características que } \\
\text { lo llevarían a elegir } \\
\text { un colegio-primera } \\
\text { mención }\end{array}$ & $\begin{array}{l}0 \text { = Motivos distintos a (1) } \\
1 \text { = Son colegios donde a } \\
\text { los alumnos les va bien } \\
\text { y en general entran a la } \\
\text { universidad }\end{array}$ & $\begin{array}{l}0=N_{0} \\
1=S_{i}\end{array}$ & $\begin{array}{l}0=\text { Signo positivo laumenta } \\
\text { probabilidad de preferir } \\
\text { EMTP) } \\
1=\text { Signo negativo (disminuye } \\
\text { probabilidad de preferir } \\
\text { EMTP) }\end{array}$ \\
\hline $\begin{array}{l}\text { Track } \\
\text { Percepción de } \\
\text { la separación } \\
\text { de alumnos en } \\
\text { el colegio según } \\
\text { habilidades }\end{array}$ & $\begin{array}{l}0 \text { = Los alumnos con } \\
\text { mayores habilidades } \\
\text { deben acceder a una } \\
\text { educación más intensiva, } \\
\text { para así poder desarrollar } \\
\text { sus capacidades } \\
1 \text { = Todos los alumnos, } \\
\text { independientemente de } \\
\text { sus capacidades, deben } \\
\text { acceder al mismo tipo de } \\
\text { educación }\end{array}$ & $\begin{array}{l}0=S i \\
1=N_{0}\end{array}$ & $\begin{array}{l}0=\text { Signo negativo (disminuye } \\
\text { probabilidad de preferir } \\
\text { EMTP) } \\
1=\text { Signo positivo laumenta } \\
\text { probabilidad de preferir } \\
\text { EMTP) }\end{array}$ \\
\hline $\begin{array}{l}\text { Nivel } \\
\text { Nivel educativo } \\
\text { necesario para } \\
\text { que el alumno } \\
\text { desempeñe el } \\
\text { trabajo que desea } \\
\text { tener }\end{array}$ & $\begin{array}{l}0 \text { = Todas las categorías, } \\
\text { excepto EMTP, como nivel } \\
\text { educativo esperado para } \\
\text { alcanzar el trabajo que el } \\
\text { estudiante desea tener } \\
1 \text { = EMTP como nivel } \\
\text { educativo esperado para } \\
\text { alcanzar dicho trabajo }\end{array}$ & $\begin{array}{l}0=N o \\
1=S i\end{array}$ & $\begin{array}{l}0=\text { Signo negativo Idisminuye } \\
\text { probabilidad de preferir } \\
\text { EMTP) } \\
1=\text { Signo positivo laumenta } \\
\text { probabilidad de preferir } \\
\text { EMTP) }\end{array}$ \\
\hline $\begin{array}{l}\text { Expectativas } \\
\text { Consideración } \\
\text { de que, en la } \\
\text { propia clase } \\
\text { lautopercibida), los } \\
\text { jóvenes van a la } \\
\text { universidad }\end{array}$ & $\begin{array}{l}0 \text { = Los jóvenes de la } \\
\text { clase autopercibida no } \\
\text { van a la universidad } \\
1=\text { Los jóvenes de la } \\
\text { clase autopercibida van a } \\
\text { la universidad }\end{array}$ & $\begin{array}{l}0=S_{i} \\
1=N_{0}\end{array}$ & $\begin{array}{l}0=\text { Signo positivo laumenta } \\
\text { probabilidad de preferir } \\
\text { EMTP) } \\
1=\text { Signo negativo (disminuye } \\
\text { probabilidad de preferir } \\
\text { EMTP) }\end{array}$ \\
\hline
\end{tabular}

Fuente: Elaboración propia sobre la base de información del proyecto PASC.

De esta forma, las categorías de las variables relacionadas con el habitus asociadas a una disposición para cursar estudios universitarios disminuirían la probabilidad de optar por un colegio con EMTP; inversamente, aquellas categorías no asociadas a una disposición a cursar estudios universitarios aumentarían la probabilidad de preferir la EMTP. 
3.4. Modelos de regresión estimados

3.4.1. Modelo prediciendo elección de modalidad educativa para la enseñanza secundaria

Para predecir la modalidad educativa en la enseñanza secundaria (establecida de manera binaria) se estimó el siguiente modelo logit:

$$
\begin{aligned}
& \operatorname{Pr}(\text { EMTP }=1) \\
& =\frac{\mathrm{e}^{\beta_{0}+\beta_{1} N S E_{\text {Bajo }}+\beta_{2} \text { Oferta }+\beta_{3} \text { Hombre }+\beta_{4} \text { Motivo }+\beta_{5} \text { Track }+\beta_{6} \text { Nivel }+\beta_{7} \text { Expectativa }+ \text { e }}}{1+\mathrm{e}^{\beta_{0}+\beta_{1} N S E_{-} \text {Bajo }+\beta_{2} \text { Oferta }+\beta_{3} \text { Hombre }+\beta_{4} \text { Motivo }+\beta_{5} \text { Track }+\beta_{6} \text { Nivel }+\beta_{7} \text { Expectativa }+e}}
\end{aligned}
$$

En la Tabla 3 se presenta la especificación de las variables incluidas: 
Tabla 3. Variables del análisis de predicción de la modalidad educativa preferida para la enseñanza media

\begin{tabular}{|c|c|c|}
\hline Dimensión & Variable & Forma de medición \\
\hline \multirow{4}{*}{$\begin{array}{l}\text { Variables } \\
\text { sociodemográficas }\end{array}$} & $\begin{array}{l}\text { Modalidad educativa } \\
\text { (EMTP) }\end{array}$ & $\begin{array}{l}\text { Variable dummy indicando colegio con EMTP } \\
\text { (categoría de referencia: colegio sin EMTP) }\end{array}$ \\
\hline & $\begin{array}{l}\text { NSE según apreciación } \\
\text { externa (NSE_bajo) }\end{array}$ & $\begin{array}{l}\text { Variable dummy indicando niveles } D \text { y E* (categoría de } \\
\text { referencia: niveles } A B C 1, C 1, C 2 \text { y C3) }\end{array}$ \\
\hline & Sexo (Hombre) & $\begin{array}{l}\text { Variable dummy indicando alumno de sexo masculino } \\
\text { (categoría de referencias: sexo femenino) }\end{array}$ \\
\hline & $\begin{array}{l}\text { Oferta académica en la } \\
\text { comuna del estudiante } \\
\text { (Oferta) }\end{array}$ & $\begin{array}{l}\text { Porcentaje de colegios con modalidad técnico- } \\
\text { profesional en la comuna del estudiante. Esta } \\
\text { variable se computó para esta investigación usando } \\
\text { información de Demre }\end{array}$ \\
\hline \multirow{4}{*}{$\begin{array}{l}\text { Variables } \\
\text { relacionadas con el } \\
\text { habitus }\end{array}$} & $\begin{array}{l}\text { Características que lo } \\
\text { llevarían a elegir un } \\
\text { colegio-primera mención } \\
\text { (Motivo): }\end{array}$ & $\begin{array}{l}\text { Variable dummy indicando la mención “Son colegios } \\
\text { donde a los alumnos les va bien y en general entran } \\
\text { a la universidad" (categoría de referencia: todas las } \\
\text { demás opciones) }\end{array}$ \\
\hline & $\begin{array}{l}\text { Percepción sobre la } \\
\text { separación según } \\
\text { habilidades de alumnos } \\
\text { (Track) }\end{array}$ & $\begin{array}{l}\text { Variable dummy indicando la afirmación: "Todos los } \\
\text { alumnos, independientemente de sus capacidades, } \\
\text { deben acceder al mismo tipo de educación" (categoria } \\
\text { de referencia: "los alumnos con mayores habilidades } \\
\text { deben acceder a una educación más intensiva, para asi } \\
\text { poder desarrollar sus capacidades") }\end{array}$ \\
\hline & $\begin{array}{l}\text { Nivel educativo necesario } \\
\text { para alcanzar el trabajo } \\
\text { que el estudiante desea } \\
\text { tener es EMTP (Nivel): } \\
\end{array}$ & $\begin{array}{l}\text { Variable dummyindicando la mención "Enseñanza media } \\
\text { técnico-profesional como el nivel educativo necesario } \\
\text { para alcanzar el trabajo que le gustaría al estudiante" } \\
\text { (categoría de referencia: todos los demás niveles) }\end{array}$ \\
\hline & $\begin{array}{l}\text { Considera que, en la } \\
\text { clase social en la que se } \\
\text { autopercibe, los jóvenes } \\
\text { van a la universidad } \\
\text { (Expectativa) }\end{array}$ & $\begin{array}{l}\text { Variable dummy indicando que los jóvenes de la clase } \\
\text { social autopercibida van a la universidad (categoría de } \\
\text { referencia: los jóvenes de la clase social autopercibida } \\
\text { no van a la universidad)** }\end{array}$ \\
\hline
\end{tabular}

Fuente: Elaboración propia sobre la base de información del proyecto PASC.

* Se optó por agrupar los niveles socioeconómicos loriginalmente: ABC1, C2, C3, D y El para contar con grupos de mayor tamaño.

** Esta variable se creó utilizando dos variables de la encuesta: en la primera se preguntó acerca de la clase autopercibida y en la segunda se indagó, para cada clase diferenciada, en diversos aspectos, incluyendo si en dicha clase los jóvenes asistían a la universidad.

\subsubsection{Modelos prediciendo la postulación a las universidades del CRUCH}

Para la predicción de la postulación a las universidades del CRUCH, se estimó el siguiente modelo:

$$
\begin{aligned}
& \operatorname{Pr}(\operatorname{Post} U=1) \\
& =\frac{\mathrm{e}^{\beta_{0}+\beta_{1} \text { StdNEM }+\beta_{2} \text { StdMat }+\beta_{3} \text { STDLeng }+\beta_{4} \text { EdMad }+\beta_{5} \text { Hombre }+\beta_{6} \text { Subv }+\beta_{7} \text { EMTP }+\beta_{8} \text { EMPV }+e}}{1+\mathrm{e}^{\beta_{0}+\beta_{1} \text { StdNEM }+\beta_{2} \text { StdMat }+\beta_{3} \text { STDLeng }+\beta_{4} \text { EdMad }+\beta_{5} \text { Hombre }+\beta_{6} \text { Subv }+\beta_{7} \text { EMTP }+\beta_{8} E M P V+e}}
\end{aligned}
$$


Los predictores corresponden tanto a variables de los estudiantes como de los establecimientos y se detallan en la Tabla 4.

Tabla 4. Variables del análisis de postulación a las universidades del CRUCH

\begin{tabular}{|c|c|c|}
\hline Dimensión & Variable & Forma de medición \\
\hline $\begin{array}{l}\text { Variable de } \\
\text { postulación }\end{array}$ & $\begin{array}{l}\text { Postulación al CRUCH } \\
\text { (PostU) }\end{array}$ & $\begin{array}{l}\text { Variable dummy indicando si el joven postula a } \\
\text { cualquiera de las universidades participantes del } \\
\text { sistema de admisión centralizado }\end{array}$ \\
\hline \multirow{2}{*}{$\begin{array}{l}\text { Variables } \\
\text { sociodemográficas }\end{array}$} & Sexo (Hombre) & $\begin{array}{l}\text { Variable dummy indicando sexo masculino (categoría } \\
\text { de referencia: sexo femenino) }\end{array}$ \\
\hline & $\begin{array}{l}\text { Nivel educativo de la } \\
\text { madre (EdMad) }\end{array}$ & $\begin{array}{l}\text { Variable categórica tratada como continua. Las categorías } \\
\text { están ordenadas de modo ascendente y van desde "sin } \\
\text { estudios" a "educación universitaria completa" }\end{array}$ \\
\hline \multirow{3}{*}{$\begin{array}{l}\text { Variables } \\
\text { académicas }\end{array}$} & $\begin{array}{l}\text { Puntaje PSU Matemática } \\
\text { (StdMat) }\end{array}$ & Variable continua (rango = 162-850) estandarizada \\
\hline & $\begin{array}{l}\text { Puntaje PSU Lenguaje y } \\
\text { Comunicación (StdLeng) }\end{array}$ & Variable continua (rango = 150-836) estandarizada \\
\hline & $\begin{array}{l}\text { Nota de enseñanza media } \\
\text { (StdNEM) }\end{array}$ & Variable continua (rango = 208-826) estandarizada \\
\hline \multirow{3}{*}{$\begin{array}{l}\text { Variables de } \\
\text { establecimiento } \\
\text { educacional }\end{array}$} & $\begin{array}{l}\text { Educación media técnico- } \\
\text { profesional (EMTP) }\end{array}$ & $\begin{array}{l}\text { Variable dummyindicando establecimiento de educación } \\
\text { media técnico-profesional (categoría de referencia: } \\
\text { colegio solo con enseñanza media científico humanista) }\end{array}$ \\
\hline & $\begin{array}{l}\text { Educación media } \\
\text { polivalente (EMPV }\end{array}$ & $\begin{array}{l}\text { Variable dummy indicando establecimiento de } \\
\text { educación media polivalente (categoría de referencia: } \\
\text { colegio solo con enseñanza media científico humanista) }\end{array}$ \\
\hline & $\begin{array}{l}\text { Establecimiento particular } \\
\text { subvencionado (Subv) }\end{array}$ & $\begin{array}{l}\text { Variable dummy indicando colegio Particular } \\
\text { subvencionado (categoría de referencia: colegio } \\
\text { municipal) }\end{array}$ \\
\hline
\end{tabular}

Fuente: Elaboración propia sobre la base de información Demre.

\subsection{Limitaciones de la investigación}

Una de las principales limitaciones de esta investigación es no contar con información longitudinal que permita seguir a una misma cohorte de estudiantes para explicar tanto la elección de modalidad educativa en la enseñanza media, como la postulación a la universidad. Asimismo, el concepto de habitus en esta investigación fue abordado indirectamente, a partir de un conjunto de variables empíricas relacionadas con las funciones que las familias asocian a la educación y a las expectativas que estas tienen de los estudiantes ${ }^{13}$. Del mismo

13 Es importante mencionar que la encuesta PASC II, utilizada para dar cuenta de este concepto, no tenía como foco específico medir el habitus, con las limitaciones que esto conlleva en términos de medición. 
modo, el análisis usado en esta investigación (regresión logística) se distancia de la aproximación relacional con la que generalmente se ha abordado el habitus y que utiliza técnicas como el análisis de correspondencias $^{14}$.

En relación con la utilización de la modalidad educativa como variable predictora, es importante destacar que la metodología utilizada no permite hacerse cargo completamente del sesgo asociado a esta variable, que tiene que ver con las particularidades de los estudiantes que optan por la EMTP. Respecto de la variable de postulación utilizada, una de sus principales limitaciones es que hace referencia solo a las universidades del $\mathrm{CRUCH}$, por lo que de existir información en el futuro respecto de la postulación al resto de las instituciones, sería muy enriquecedor incorporarla en los análisis. Abordar la decisión de postulación cuenta además con una complejidad especial: es difícil distinguir qué está detrás de la decisión de postular o no a la universidad. Si bien en algunos casos puede tratarse de falta de interés o de incumplimiento de requisitos académicos, en otros, puede deberse a motivos muy distintos, como la falta de información acerca de la oferta de programas e instituciones o la inseguridad de los postulantes respecto de sus posibilidades de ser admitidos.

\section{Resultados}

\subsection{Elección de modalidad educativa en la enseñanza media}

\subsubsection{Análisis descriptivo}

Se analizó la relación entre las variables vinculadas con el habitus utilizadas en esta investigación y la modalidad educativa de la primera preferencia de establecimiento para la enseñanza media, observándose diferencias en la línea de lo hipotetizado entre quienes optan por colegios con EMTP y quienes prefieren colegios que ofrecen exclusivamente EMCH (Tabla 5).

14 No obstante, cabe destacar que una de las debilidades del análisis de correspondencia, es que si bien permite mostrar relaciones entre variables, no se puede testear su nivel de ajuste, algo que sí ofrecen los modelos de regresión. 
Tabla 5. Variables relacionadas con el habitus y modalidad educativa del establecimiento preferido por las familias

\begin{tabular}{|c|c|c|c|}
\hline Variables relacionadas con el habitus & $\begin{array}{c}\text { Preferencia por } \\
\text { establecimiento } \\
\text { EMCH }\end{array}$ & $\begin{array}{c}\text { Preferencia por } \\
\text { establecimiento } \\
\text { EMTP } \\
\end{array}$ & Total \\
\hline \multicolumn{4}{|l|}{$\begin{array}{l}\text { Principal motivo de preferencia del establecimiento } \\
\text { (variable motivo) }\end{array}$} \\
\hline \multirow{2}{*}{ Motivo académico (permite ingresar a la universidad) } & $30,9 \%$ & $23,3 \%$ & $26,5 \%$ \\
\hline & 46 & 48 & 94 \\
\hline \multirow{2}{*}{ Otros aspectos* } & $69,1 \%$ & $76,7 \%$ & $73,5 \%$ \\
\hline & 103 & 158 & 261 \\
\hline \multicolumn{4}{|l|}{$\left(\mathrm{Chi}^{2}=2,54 ; \mathrm{Pr}=0,1\right)$} \\
\hline \multicolumn{4}{|l|}{ Preferencia por tracking académico (variable tracking) } \\
\hline \multirow{2}{*}{$\begin{array}{l}\text { Preferencia por entregar la misma educación a todos los } \\
\text { estudiantes }\end{array}$} & $51,7 \%$ & $65,5 \%$ & $59,7 \%$ \\
\hline & 77 & 135 & 212 \\
\hline \multirow{2}{*}{ Preferencia por diferenciar según habilidad académica } & $47,0 \%$ & $34,0 \%$ & $39,4 \%$ \\
\hline & 70 & 70 & 140 \\
\hline \multirow{2}{*}{ NS/NR } & $1,3 \%$ & $0,5 \%$ & $0,9 \%$ \\
\hline & 2 & 1 & 3 \\
\hline \multicolumn{4}{|l|}{$\left(C h i^{2} \sin N S / N R=6,48 ; \operatorname{Pr}=0,01\right)$} \\
\hline \multicolumn{4}{|l|}{$\begin{array}{l}\text { Nivel educativo esperado para el estudiante /variable } \\
\text { nivel) }\end{array}$} \\
\hline \multirow{2}{*}{ Media técnico-profesional } & $2,7 \%$ & $11,7 \%$ & $7,9 \%$ \\
\hline & 4 & 24 & 28 \\
\hline \multirow{2}{*}{$\begin{array}{l}\text { Otros niveles educativos (media científico humanista, } \\
\text { universitaria, técnico-profesional, posgrado) }\end{array}$} & $91,3 \%$ & $81,0 \%$ & $85,3 \%$ \\
\hline & 136 & 167 & 303 \\
\hline \multirow{2}{*}{ NS/NR } & $6,0 \%$ & $7,3 \%$ & $6,8 \%$ \\
\hline & 9 & 15 & 24 \\
\hline \multicolumn{4}{|l|}{ (Chi²=9,57 sin NS/NR; Pr = 0,002) } \\
\hline \multicolumn{4}{|l|}{$\begin{array}{l}\text { Jóvenes de su clase social van a la universidad (variable } \\
\text { expectativa) }\end{array}$} \\
\hline \multirow{2}{*}{ Jóvenes de su clase van a la universidad } & $59,1 \%$ & $36,9 \%$ & $53,8 \%$ \\
\hline & 88 & 76 & 164 \\
\hline \multirow{3}{*}{ Jóvenes de su clase no van a la universidad } & $40,9 \%$ & $63,1 \%$ & $46,2 \%$ \\
\hline & & & \\
\hline & 61 & 130 & 191 \\
\hline \multicolumn{4}{|l|}{$\left(\mathrm{Chi}^{2}=17,09 ; \mathrm{Pr}=0,000\right)$} \\
\hline Total general de casos & 149 & 206 & 355 \\
\hline
\end{tabular}

Fuente: Elaboración propia sobre la base de información del proyecto PASC.

Se observa una menor proporción de quienes declaran una motivación académica en la preferencia de establecimiento de enseñanza media entre quienes prefieren un colegio con EMTP. Asimismo, entre quienes mencionan un colegio con EMTP como 
primera preferencia, existe una menor proporción de familias que dicen preferir una diferenciación de estudiantes según habilidad académica en comparación con el grupo que opta por un establecimiento EMCH. Por otra parte, en el caso de quienes prefieren un colegio con EMTP para la enseñanza secundaria, se observa una mayor proporción de familias que considera que la enseñanza media técnico-profesional es la adecuada para sus hijos, dado el trabajo que estos quieren ejercer ${ }^{15}$. Por último, existe un menor porcentaje de familias que cree que los jóvenes de su clase social autopercibida van a la universidad entre quienes prefieren un colegio con EMTP, en comparación con quienes prefieren un colegio que ofrezca exclusivamente EMCH.

\subsubsection{Análisis de regresión}

La Tabla 6 muestra los resultados del modelo estimado para la predicción de modalidad educativa preferida para la enseñanza media. En la columna de la izquierda se presenta el modelo restringido (sin las variables relacionadas con el habitus) y en la columna de la derecha se presenta el modelo completo (incluyendo las variables relacionadas con el habitus). La interpretación se hará para el modelo completo ${ }^{16}$.

15 Entre quienes prefieren un colegio con EMTP, solo alrededor de un $12 \%$ considera este nivel como el nivel final a alcanzar por parte del estudiante (un 51,5\% considera que el nivel a alcanzar debería ser el universitario).

16 Además de observar un mayor Pseudo $\mathrm{R}^{2}$ en el caso del modelo completo, para conocer el cambio en el ajuste de los modelos gracias la introducción de las variables relacionadas con el habitus se realizó un Likelihood-ratio test (lr test) con los dos modelos estimados. Los resultados del test indican una diferencia significativa a un 99\% de confianza (lr test: 20,12) entre ambas especificaciones, es decir, que la introducción de las variables relacionadas con el habitus mejora el ajuste del modelo para la predicción de la modalidad educativa preferida en la enseñanza secundaria. El test AIC (modelo restringido: 441 y modelo completo: 429), apunta en la misma línea, indicando mejor ajuste para el segundo modelo, en tanto que el test BIC establece como mejor modelo el restringido (modelo restringido: 456 y modelo completo: 460). Pese a este último resultado, se puede afirmar que es pertinente incorporar las variables relacionadas con el habitus en el modelo y, en específico, las variables de motivo de la elección (motivo) y de nivel educativo EMTP como el esperado para el joven (nivel), ya que permiten un mejor ajuste del modelo según dos de los test realizados. 
Tabla 6. Modelo de regresión logística prediciendo la preferencia de modalidad educativa para la enseñanza media

\begin{tabular}{l|c|c}
\hline Variables & Modelo restringido & Modelo completo \\
\hline Oferta & $0,566^{* * *}$ & $0,494^{* * *}$ \\
\hline NSE Bajo & $(-0,139)$ & $(-0,142)$ \\
\hline & $0,890^{* * *}$ & $0,797^{* * *}$ \\
\hline Hombre & $(-0,242)$ & $(-0,26)$ \\
\hline & $-0,000622$ & $-0,0427$ \\
\hline Motivo & $(-0,231)$ & $(-0,24)$ \\
\hline & & $-0,600^{* *}$ \\
\hline Track & & $(-0,27)$ \\
\hline & & 0,383 \\
\hline Nivel & & $(-0,244)$ \\
\hline & & $1,319 * *$ \\
\hline Expectativa & & $(-0,58)$ \\
\hline & & $-0,351$ \\
\hline Constante & $-0,0513$ & $(-0,257)$ \\
\hline & $(-0,209)$ & $-0,03$ \\
\hline Observaciones & 355 & $(-0,315)$ \\
\hline Pseudo R & & 352 \\
\hline Fente:EForion & 0,104 & 0,137 \\
\hline
\end{tabular}

Fuente: Elaboración propia sobre la base de información Proyecto PASC.

Coeficientes no estandarizados. Errores estándares entre paréntesis.

${ }^{* * *} p<0.01,{ }^{* *} p<0.05,{ }^{*} p<0.1$.

Los resultados muestran una relación positiva y significativa entre el índice de establecimientos con EMTP de la comuna y la probabilidad de preferir como primera opción un establecimiento con EMTP para la enseñanza secundaria. También se observa una relación positiva entre la preferencia por un establecimiento con EMTP y la pertenencia a un nivel socioeconómico bajo (segmento D o E). En tanto, la variable sexo no resulta significativa.

Solo dos de las variables relacionadas con el habitus resultaron significativas: el principal motivo de la preferencia por el establecimiento (motivo) y aquella que hace referencia a la EMTP como nivel educativo esperado para el alumno según el trabajo que el joven desea tener (nivel). En específico, los resultados de la regresión indican 
que si la dimensión académica ("son colegios donde a los alumnos les va bien y en general entran a la universidad") es el principal motivo en la preferencia por el establecimiento educacional para la enseñanza secundaria, existe una menor probabilidad de optar un colegio EMTP: quienes declaran el motivo académico como el principal en la preferencia por el establecimiento de enseñanza media tienen alrededor de la mitad de las chances (55\%) de optar por un colegio con EMTP que quienes no consideran este motivo como el principal en la preferencia de establecimiento. Por otro lado, que el EMTP sea el nivel educativo esperado para el estudiante dado el trabajo que este quiere desempeñar (variable nivel) está asociado con una mayor probabilidad de optar por un colegio con EMTP: considerar este nivel como el esperado para el estudiante aumenta en un $274 \%$ las probabilidades de preferir un colegio con EMTP. El resto de los coeficientes de las variables relacionadas con el habitus no resultó significativo en los niveles convencionales

\subsection{Postulación a las universidades del CRUCH}

\subsubsection{Análisis descriptivo}

En la Tabla 7 se presentan los totales y la distribución por modalidad educativa de quienes rinden la PSU y de quienes postulan a algunas de las universidades del CRUCH.

Tabla 7. Postulación a las universidades del CRUCH según modalidad educativa (admisión 2011)

\begin{tabular}{l|c|c|c|c|c|c}
\hline Proceso 2011 & EMCH & EMTP & EMPV & Válidos & Perdidos & Total \\
\hline Inscritos del proceso & 98.043 & 52.081 & 37.200 & 187.324 & 24.367 & 211.691 \\
\hline Rinden la PSU & 92.257 & 40.412 & 30.666 & 163.335 & 15.913 & 179.248 \\
\hline \begin{tabular}{l} 
(\% rinde PSU sobre total inscritos) \\
\hline Postulan al CRUCH
\end{tabular} & $94,10 \%$ & $77,60 \%$ & $82,40 \%$ & $87,20 \%$ & $65,30 \%$ & $84,70 \%$ \\
\hline $\begin{array}{l}\text { (\% postulan al CRUCH sobre total } \\
\text { que rinde) }\end{array}$ & 44.113 & 67.225 & 7.227 & 57.565 & 2.044 & 59.609 \\
\hline
\end{tabular}

Fuente: Elaboración propia sobre la base de información Demre.

Tanto el total de jóvenes inscritos, como el de quienes rinden y postulan a las universidades del CRUCH, varía según la modalidad educativa del colegio de egreso del estudiante, siendo menor el 
porcentaje de jóvenes provenientes de la EMTP y de la EMPV que participa de las distintas etapas de postulación a las universidades del $\mathrm{CRUCH}^{17}$.

\subsubsection{Análisis de regresión}

La Tabla 8 presenta los resultados del modelo prediciendo la postulación a las universidades del $\mathrm{CRUCH}$, incluyendo tanto el modelo completo como el reducido (aquel sin las variables de interés sobre modalidad educativa ${ }^{18}$ ). Cabe destacar que los valores perdidos de las variables modalidad educativa y educación de la madre hacen disminuir de manera relevante la cantidad de casos considerados en la regresión. Para dar cuenta de la naturaleza anidada de los datos (alumnos agrupados en establecimientos), se establecieron clúster de varianza a partir de los identificadores de los colegios (RBD $)^{19}$.

Tabla 8. Modelo de regresión logística prediciendo la postulación a las universidades del $\mathrm{CRUCH}$

\begin{tabular}{l|c|c}
\hline Variables & Modelo restringido & Modelo completo \\
\hline StdNEM & $0,528^{* * *}$ & $0,575^{* * *}$ \\
\hline & $(-0,02)$ & $(-0,0217)$ \\
\hline StdMat & $1,283^{* * *}$ & $1,224^{* * *}$ \\
\hline & $(-0,0249)$ & $(-0,0268)$ \\
\hline StdLeng & $0,880 * * *$ & $0,831 * * *$ \\
\hline & $(-0,0211)$ & $(-0,0218)$ \\
\hline EdMad & $0,0525 * * *$ & $0,0268^{* * *}$ \\
\hline & $(-0,00656)$ & $(-0,062)$ \\
\hline
\end{tabular}

17 No se pudo asignar modalidad educativa a todos los inscritos en el proceso, debido a la falta o inconsistencia de identificador de colegio (RBD). Asimismo, quienes se encontraban en modalidad científico-humanista en régimen nocturno no fueron considerados en el análisis, al tratarse de un grupo con características muy distintas a las del resto de los estudiantes.

18 Respecto de los estadísticos de ajuste para comparar ambos modelos, los valores de AIC (107.245 para el modelo restringido y 101.179 para el modelo completo) y BIC (107.314 para el modelo restringido y 101.267 modelo completo). sugieren que el modelo con las variables de modalidad educativa ajusta mejor que uno que no considere estas variables. Si bien no es recomendable realizar $l r$ test con datos ordenados en clúster, el resultado de este va en la misma dirección de los estadísticos AIC y BIC y se mantiene igual con los modelos sin considerar clúster de varianza, indicando con un $99 \%$ de confianza que es preferible el modelo que incluye las variables de modalidad educativa (lr test: 6.070,76).

19 Dichos clúster generan errores estándares robustos, pudiendo afectar la significancia de los coeficientes. 


\begin{tabular}{l|c|c}
\hline Hombre & $-0,0666^{* * *}$ & $-0,0152$ \\
\hline Subv & $(-0,0308)$ & $(-0,0313)$ \\
\hline & $-0,105^{* *}$ & $-0,168^{* * *}$ \\
\hline EMTP & $(-0,058)$ & $(-0,0603)$ \\
\hline & & $-0,558^{* * *}$ \\
\hline EMPV & & $(-0,0756)$ \\
\hline Constante & & $-0,207 * * *$ \\
\hline & $-1,176^{* * *}$ & $(-0,0647)$ \\
\hline Observaciones & $(-0,0541)$ & $-0,851 * * *$ \\
\hline Pseudo R & 139.317 & $(-0,0661)$ \\
\hline
\end{tabular}

Fuente: Elaboración propia sobre la base de información Demre.

Coeficientes no estandarizados. Errores estándares entre paréntesis.

${ }^{* * *} p<0.01,{ }^{* *} p<0.05,{ }^{*} p<0.1$.

Los resultados del modelo completo muestran que los coeficientes relacionados con los criterios de admisión (puntaje NEM y puntajes PSU Matemática y Lenguaje) tienen la dirección esperada: a medida que aumentan estos puntajes, se incrementan las probabilidades de postular a las universidades del CRUCH. El nivel educativo de la madre también se relaciona de manera positiva con la probabilidad de postular al CRUCH, en tanto que el haber egresado de un establecimiento subvencionado disminuye dichas probabilidades, manteniendo el resto de las variables constantes. El coeficiente de la variable de sexo no resultó significativo.

Las variables de interés de la investigación (EMTP y EMPV) presentan un coeficiente negativo lo que indica que, manteniendo todo lo demás constante, el haber asistido a un establecimiento con EMTP o EMPV, en vez que a uno con EMCH, disminuye las probabilidades de postular a las universidades del CRUCH. En particular, los valores en términos de odds ratio muestran que las chances de postular a una universidad del CRUCH habiendo egresado de un establecimiento EMTP corresponden a un poco más de la mitad (57\%) de las que tienen quienes egresaron de la $\mathrm{EMCH}$, mientras que quienes egresaron de la EMPV tienen un $81 \%$ de las chances de los graduados de la EMCH de postular a las universidades del $\mathrm{CRUCH}$. 


\section{Discusión}

Los resultados de la presente investigación muestran que la elección de modalidad educativa para la enseñanza media está asociada a variables sociodemográficas, en particular, nivel socioeconómico y oferta educativa en la comuna, lo que va de la mano de los antecedentes recabados por la literatura nacional (De Iruarrizaga, 2009; Larrañaga et al., 2013). En tanto, esta investigación no entrega evidencia suficiente para sustentar la hipótesis de la importancia de todas las variables relacionadas con el habitus abordadas en la predicción de elección de modalidad educativa, resultando significativas solo dos de ellas: motivo académico de la preferencia del establecimiento de enseñanza secundaria (variable motivo) y EMTP como el nivel educativo esperado para el estudiante según el trabajo que este quiere desempeñar (variable nivel).

En relación con la segunda hipótesis, esta investigación encontró que las variables académicas y socioeconómicas están positivamente relacionadas con la postulación a las universidades del $\mathrm{CRUCH}$, resultado que es consistente con la literatura anterior (Cabrera \& La Nasa, 2001). Por su parte, el haber asistido a un colegio particular subvencionado en vez que a uno municipal disminuye la probabilidad de postular al $\mathrm{CRUCH}$, manteniendo todas las demás variables constantes. Las variables de modalidad educativa resultan significativas y negativamente asociadas a la predicción de la postulación a las universidades del CRUCH. Esto indica que, aun controlando por el resto de las variables, incluyendo la habilidad académica, el haber egresado de establecimientos EMTP y EMPV disminuye las probabilidades de postular a las universidades tradicionales del país.

Los resultados anteriormente discutidos evidencian que el contexto socioeconómico afecta las decisiones educativas a partir de una serie de mecanismos, incluyendo la disponibilidad de recursos financieros e institucionales, la oferta local de establecimientos, pero también, las funciones que las familias asocian con la educación y las expectativas educacionales que tienen de sus hijos. La significancia estadística de dos de las variables relacionadas con el habitus, incluso 
al controlar por nivel socioeconómico, alienta a considerar dentro de la investigación de las decisiones educativas cómo las condiciones objetivas de los individuos se traducen en disposiciones que dan el marco general para sus decisiones y prácticas (Bourdieu, 1993). De la misma manera, la importancia de la modalidad educativa cursada en la enseñanza secundaria en la predicción de la postulación a las universidades del CRUCH respalda la hipótesis de la relevancia de esta modalidad sobre las trayectorias hacia la educación superior, en forma adicional a aspectos relacionados con las habilidades académicas (puntajes PSU y NEM).

En su conjunto, los resultados de esta investigación entregan evidencia respecto del rol que tendría el tracking académico durante la educación secundaria en la reproducción de las inequidades sociales y educativas, como ha destacado la literatura internacional (Hallinan, 1994) y nacional (Farías, 2013). En este marco, surge la pregunta acerca de la pertinencia de la diferenciación temprana de estudiantes en tracks académicos y laborales, especialmente al tomar en cuenta el creciente interés que tienen los jóvenes que cursan la EMTP por continuar sus estudios una vez finalizada la enseñanza secundaria. Sin duda, es un tema que ha sido poco explorado por la literatura nacional, por lo que se espera que este estudio haya entregado antecedentes valiosos para la discusión, además de incentivar la futura investigación al respecto.

En futuras investigaciones sería enriquecedor contar con datos longitudinales que permitan hacer seguimiento a una cohorte en específico a lo largo de su trayectoria a la educación superior, al igual que el uso de técnicas más avanzadas para la estimación de efectos causales. Junto con esto, se sugiere abordar el concepto de habitus de una manera más completa, e integrando preguntas específicas en relación con la diferenciación entre modalidades educativas y las consideraciones de las familias y de los jóvenes al respecto. En este contexto, la utilización de metodología cualitativa podría ser muy enriquecedora.Asimismo, incluir variables relacionadas con la motivación y con las expectativas de los estudiantes y sus familias respecto del futuro académico de los jóvenes podría mejorar la especificación de los modelos para medir el aporte de la modalidad 
educativa de enseñanza media a las trayectorias educacionales, En estudios futuros también podrían hacerse distinciones más finas dentro de las modalidades educativas, diferenciando entre especialidades de la EMTP o incluyendo variables relacionadas con la calidad académica de los establecimientos, procesos de selección de estudiantes, etc.

\section{Referencias}

Acuña, C., Makovec, M., \& Mizala, A. (2010, octubre). Access to higher education and dropouts: Evidence from a cohort of Chilean secondary school leavers. Trabajo presentado en el Congreso Interdisciplinario de Investigación en Educación, Santiago, Chile.

Adelman, C. (2006). The toolbox revisited: Paths to degree completion from high school through college. Washington, D.C.: U.S. Department of Education.

Bell, A., Rowan-Kenyon, H., \& Perna, L. (2006). College knowledge of 9th and 11th grade students: Variation by school and state context. The Journal of Higher Education, 80(6), 663-685. http://dx.doi.org/10.1353/ jhe.0.0074

Bourdieu, P. (1984). Distinction: A social critique of the judgment of taste. Cambridge, MA: Harvard University Press.

Bourdieu, P. (1987). Habitus, code, codification. Actes de la Recherche en Sciences Sociales, 64, 17.

Bourdieu, P. (1993). El sentido práctico. Madrid: Taurus.

Bourdieu, P. (1997). Razones prácticas: sobre la teoría de la acción. Barcelona: Anagrama.

Bourdieu, P. (2001). Las formas del capital. En P. Bourdieu, Poder, derecho y clases sociales (pp. 131-164). Bilbao: Desclée de Brouwer.

Bourdieu, P. (2006). Argelia 60: estructuras económicas y estructuras temporales. Buenos Aires: Siglo XXI.

Cabrera, A. \& La Nasa, S. (2001). On the path to college. Three critical tasks facing America's disadvantaged. Research in Higher Education, 42(2), 119-149. http://dx.doi.org/10.1023/A:1026520002362

Canales, A. (abril, 2015). Diferencias socioeconómicas en la postulación y postulaciones a las universidades chilenas. Trabajo presentado en las Tertulias de Educación Superior (TES), Santiago, Chile.

Centro de Estudios del Ministerio de Educación de Chile, Mineduc. (2013). Educación media técnico profesional: hallazgos del seguimiento a una generación. Serie Evidencias, año 2, No 20. Santiago: Autor. 
Comisión de financiamiento estudiantil para la educación superior en Chile (2012). Análisis y recomendaciones para el sistema de financiamiento estudiantil. Recuperado de http://www.mineduc.cl/usuarios/mineduc/ doc/201203291032500.InformeComisionAyudaEstudiantilmar zo2012.pdf

Consejo Nacional de Educación, CNED. (2010). Estadísticas de cohorte por institución. Recuperado de http://www.cned.cl/public/Secciones/ SeccionIndicesEstadisticas/indices_estadisticas_retencion.aspx

Demre (2011). Compendio estadístico. Proceso de admisión año académico 2011. Recuperado de http://www.psu.demre.cl/estadisticas/documentos/ p2011/2011-compendio-estadistico.pdf

Dumais, S. (2002). Cultural capital, gender, and school success: The role of habitus. Sociology of Education, 75(1), 44-68. http://dx.doi. org/10.2307/3090253

Elacqua, G. y Fabrega, R. (2004). El consumidor de la educación: el actor olvidado de la libre elección de escuelas en Chile. Santiago: Universidad Adolfo Ibáñez.

Farías, M. (2013). Effects of early career decisions on future opportunities: The case of vocational education in Chile (Tesis para optar al grado de doctor, Universidad de Stanford, California, EE.UU.). Recuperado de http:// purl.stanford.edu/cr356cg6697

Farías, M. y Carrasco, R. (2012). Diferencias en resultados académicos entre la educación media técnico-profesional y humanista-científica en Chile. Revista Calidad en la Educación, 36, 87-121. http://dx.doi.org/10.4067/ s0718-45652012000100003

Farías, M. y Sevilla, M. P. (2012). Efectividad de la enseñanza media técnicoprofesional en la persistencia y rendimiento en la educación técnica superior. Recuperado de http://static.mineduc.cl/doc_mailing/ efectividad $\% 20$ de $\% 20$ la $\% 20$ emtp $\% 20$ en $\% 20$ la $\% 20$ persistencia $\% 20$ y\%20rendimiento\%20en\%20la\%20estp.pdf

Flores, C. y Carrasco, A. (2013). (Des)igualdad de oportunidades para elegir escuela: preferencias, libertad de elección y segregación escolar. Recuperado de http://espaciopublico.cl/publicaciones/detalle.tpl?id=8

Hallinan, M. T. (1994). School differences in tracking effects on achievement. Social Forces 72(3), 799-820. http://dx.doi.org/10.1093/sf/72.3.799

Hossler, D., Braxton, J., \& Coopersmith, G. (1989). Understanding student college choice. En J. Smart (Ed.), Higher education: Handbook of theory and research, Vol. 5 (pp. 231-288). New York: Agathon. 
De Iruarrizaga, F. (2009). Dos miradas a la educación media en Chile. Santiago: Instituto de Economía de la Pontificia Universidad Católica de Chile.

Karen, D. (1988). Who applies where to college: An empirical examination of the social context of ambition. Trabajo presentado en el Encuentro anual de la American Educational Research Association, New Orleans.

Larrañaga, O., Cabezas, G. y Dussaillant, F. (2013). Estudio de la educación técnico profesional. Recuperado de http://www.pnud.cl/areas/ ReduccionPobreza/2013/pdf_EMTP/Estudio_EMTP_PNUD.pdf

McDonough, P. (1997). Choosing colleges: How social class and schools structure opportunity. Albany: State University of New York Press.

McDonough, P. (1988). Classmates? How students prepare for different college futures. Trabajo presentado en el Encuentro anual de la American Educational Research Association, New Orleans.

Meneses, F. \& Blanco, C. (2010). Financial aid and higher education enrollment in Chile: A government policy analysis. Recuperado de http://mpra. ub.uni-muenchen.de/23321/

Ministerio de Educación de Chile, Mineduc. (2011). Educación técnico profesional en Chile: antecedentes y claves de diagnóstico. Santiago: Centro de Estudios Mineduc.

Organización para la Cooperación y el Desarrollo Económicos, OCDE. (2009). La educación superior en Chile. Revisión de politicas nacionales de educación. Santiago: Mineduc.

Pearson. (2013). Final report evaluation of the Chile PSU. Recuperado de http://www.mineduc.cl/usuarios/mineduc/doc/201301311057540. Chile_PSU-Finalreport.pdf

Perna, L. W. (2006). Studying college access and choice: A proposed conceptual model. En J. C. Smart (Ed.), Higher education: Handbook of theory and research, Vol. 21 (pp. 99-157). Netherlands: Springer.

Perna, L. W., Rowan-Kenyon, H. T., Bell, A., Thomas, S. L., \& Li, C. (2008). A typology of federal, state, and local public policies designed to promote college access. Journal of Higher Education, 79(3), 243-267. http://dx.doi.org/10.1353/jhe.0.0002

Raczynski, D., Hernández, M. Rejevic, L., y Roco, R. (2011). El paso de la enseñanza básica a la media en estratos bajos: un reto a la igualdad de oportunidades (Proyecto FONIDE). Recuperado de http://www. comunidadescolar.cl/documentacion/FONIDE/Informe\%20FinalDagmar\%20Raczynski-F511083.pdf 
Sepúlveda, L., Ugalde, P., y Campos, F. (2009). Estado y perspectivas de la enseñanza media técnico-profesional en Chile: un estudio sobre las orientaciones estratégicas predominantes en los actores (Proyecto FONIDE). Recuperado de http://sgdce.mineduc.cl/descargar.php?id_ doc $=201208211212020$

Solis, A. (2012). Does higher education cause political participation?: Evidence from a regression discontinuity design. Working Paper Series 2013:13, Uppsala University, Department of Economics.

Vester, M. (2003). Class and culture in Germany. Sociologia, Problemas e Práticas, 42, 25-64. Recuperado de http://www.scielo.mec.pt/scielo. php?script=sci_arttext\&pid=S0873-65292003000200003\&lng=en \&tlng=en.

Zwick, R. \& Grief Greene, J. (2007). New perspectives on the correlation of SAT scores, high school grades and socioeconomic factors. Journal of Educational Measurement, 44(1), 23-45. http://dx.doi.org/10.1111/ j.1745-3984.2007.00025.x

Recibido: 31/08/2015

Aceptado: 22/08/2015 